\title{
EFECTO DE Arachis pintoi SOBRE LAS ARVENSES ASOCIADAS AL PLÁTANO MACHO (Musa AAB), CÁRDENAS, TABASCO, MÉXICO1
}

\author{
Eder Ramos-Hernández ${ }^{2}$, Ángel Sol-Sánchez $z^{2}$,Armando Guerrero-Peña ${ }^{2}$, José Jesús Obrador-Olán², \\ Eugenio Carrillo-Ávila ${ }^{3}$
}

\section{RESUMEN}

Efecto de Arachis pintoi sobre las arvenses asociadas al plátano macho (Musa AAB), Cárdenas, Tabasco, México. Este experimento se realizó en Cárdenas, Tabasco, México en un suelo con textura franca, $\mathrm{pH}$ moderadamente ácido, contenido de materia orgánica y nitrógeno total bajo, cultivado con plátano macho, con el objetivo de determinar el control de arvenses con $A$. pintoi en una plantación de plátano macho. Se utilizó un arreglo factorial alojado en un diseño de bloques completos al azar, con sombreado y tipo de cobertura vegetal asociada al plátano como factores, con tres repeticiones (octubre 2008-agosto 2009). Tanto el factor sombreado como el factor cobertura tuvieron dos niveles de porcentaje: $45(\mathrm{~s}= \pm$ $11,1)$ y $50(s= \pm 13,2) \%$ de sombra, para suelos con arvenses y, con $A$. pintoi, respectivamente. Se utilizaron los índices de Shannon-Wiever $\left(H^{\prime}\right)$ y similitud de Sörensen $(S)$ para determinar la riqueza de especies. A los once meses después de establecido el experimento los valores de $H^{\prime}$ no mostraron diferencias significativas entre los tratamientos, $S=0,8$ entre los dos niveles de sombra. Las especies arvenses dominantes fueron: T. triangulare, $S$. podophylum, $P$. paniculatum y $C$. diffusa. Entre los tratamientos probados, el de suelo con arvenses a nivel de $50 \%$ de sombra fue el que presentó $(\mathrm{p}=0,05)$ mayor producción MS $\left(375,3 \pm 83,3 \mathrm{~g} / \mathrm{m}^{2}\right)$. La MS producida por A. pintoi fue mayor $(\mathrm{p}=0,05)$ con $45 \%(\mathrm{~s}= \pm 11,1)$ de sombra $\left(198 \mathrm{~g} / \mathrm{m}^{2}\right)$ que la producida a $50 \%(\mathrm{~s}= \pm 13,2)$ sombra $\left(150,4 \mathrm{~g} / \mathrm{m}^{2}\right)$. Se encontró que el nivel de efectividad de $A$. pintoi para controlar arvenses fue de 52,3\% (con $45 \pm 11,1 \%$ de sombra) y $70,5 \%$ (con $50 \pm 13,2 \%$ sombra).

\begin{abstract}
Effect of Arachis pintoi on weed control associated with plantain (Musa AAB), Cardenas, Tabasco, Mexico. The experiment was carried out in Cárdenas, Tabasco, Mexico, in a soil with loamy texture, $\mathrm{pH}$ moderately acidic, low content of organic matter and total nitrogen, and cultivated with plantain. The objective was to evaluate the effect of Arachis pintoi as a cover crop for weed control in plantain crops. The experimental design was factorial, housed in a randomized complete block, with shading and type of plantain plant cover as associated factors. Three replications were used (October 2008-August 2009). Both, the shading and the cover factors had two levels: 50 ( $\mathrm{s}= \pm$ $13,2)$ and $45 \%(s= \pm 11.1)$, and the ground was covered by weeds and by $A$. pintoi respectively. Shannon-Wiever $\left(H^{\prime}\right)$ and Sörensen $(S)$ indexes were used to determine species richness. Eleven months after the establishment of the experiment, $H^{\prime}$ values did not differ significantly, $S=0.8$, in both shade percentages. The most common weed species were $T$. triangulare, $S$. podophylum, $P$. paniculatum and $C$. diffusa. The treatment with weeds and $50 \%$ of shade had statically the highest value of MS $\left(375.3 \pm 83.3 \mathrm{~g} / \mathrm{m}^{2}\right)$. MS produced by $A$. pinto $i$ was statically higher $(\mathrm{p}=0,05)$ under $45 \%\left(198 \mathrm{~g} / \mathrm{m}^{2}\right)$ than under $50 \%$ shadea $\left(150,4 \mathrm{~g} / \mathrm{m}^{2}\right)$. The effectiveness of $A$. pintoi in weed control was $52.3 \%$ under $45 \%$ shade, and $70.5 \%$ under $50 \%$ shade, respectively.
\end{abstract}

Key words: Solar radiation, weeds, heliophytes, cover crops, Shannon-Wiever.

Palabras claves: Radiación solar, arvenses, heliófilas, cobertura viva, Shannon-Wiever.

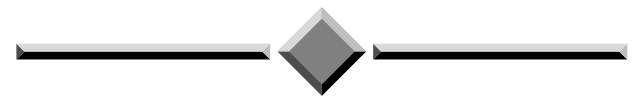

\footnotetext{
Recibido: 26 de enero, 2010. Aceptado: 30 de mayo, 2011. Parte de la tesis de grado de Maestría en Producción Agroalimentaria en el Trópico, Colegio de Postgraduados, Campus Tabasco. México.

2 Colegio de Postgraduados, Campus Tabasco. Apdo. Postal No. 24. C.P.86500. H. Cárdenas, Tabasco, México. eramos@colpos.mx; sol@ colpos.mx; garmando@colpos.mx; obradoro@colpos.mx

3 Colegio de Postgraduados, Campeche, México. ceugenio@colpos.mx
} 


\section{INTRODUCCIÓN}

Las malezas o arvenses se distinguen de las plantas silvestres que crecen en los campos agrícolas por su efecto nocivo hacia los cultivos, por lo que se cree que deberían ser eliminadas por completo de estos campos para evitar reducir el rendimiento y calidad de las cosechas, lo que causa grandes pérdidas económicas al agricultor y ambientales a los agroecosistemas (Marzocca 1979, Espinoza y Sarukhan 1997, van Rijn 2000, Gliessman 2002, Naylor 2002, Caamal 2004).

En plantaciones de plátano macho, las arvenses se consideran un problema de primer orden, ya que intervienen dentro del equilibrio ecológico provocando: problemas fitosanitarios, lento crecimiento, retraso de la floración, bajos rendimientos; y sus daños son cuantificables solamente al momento de la cosecha (Pinilla y García 2002). Por ello se deben controlar en los primeros seis meses después de la siembra y durante todo el ciclo del cultivo (Labrada 2000, van Rijn 2000, Pinilla y García 2002, Palencia et al. 2006).

El productor controla arvenses basándose en su tamaño, lo cual no necesariamente refleja la real competencia por nutrientes, como el fósforo $(\mathrm{P})$, debido a que las arvenses absorben y concentran mayor $\mathrm{P}$ que otras especies en la misma cantidad de biomasa (Muñoz 1985). Por otro lado, las que acumulan más $\mathrm{N}$ en su biomasa compiten de igual manera que otras que no contienen este nutriente en su biomasa (Muñoz 1985).

Se han desarrollado diferentes estrategias de control que tienen efectos sobre la dinámica de las arvenses-sistemas agrícolas en conjunto (Caamal 2004) y las invenciones modernas aparecidas en la segunda mitad del siglo XX, con relación al aumento de la producción agrícola fueron las sustancias químicas en el control de plagas y arvenses de los cultivos (Rojas 1980, Gliessman 2002).

El uso de herbicidas presenta ventajas comparado con el control mecánico-manual, debido a: oportunidad y persistencia del control, disminución de requerimientos de mano de obra, control eficiente de arvenses perennes, menor costo en relación al salario mínimo de la región, menor fatiga del operario, se evita la diseminación de semillas y se causa menor daño a los puyones de plátano; en contra tiene la alteración de las características físicas, químicas y biológicas de los suelos, el riesgo asociado al manejo de los productos, contaminación ambiental, posibilidad de generar resistencia de los arvenses por la utilización intensiva de herbicidas, requerimiento de un equipo costoso para las aplicaciones y posible intoxicación para el aplicador (Pinilla y García 2002).

Los métodos de control manual influyen en la población de arvenses, ya que su incidencia se reduce con el método de control químico, en comparación con el manual y sin control. Algunas arvenses se ven favorecidas por el deshierbe e incrementan en cuanto a riqueza de especies y equidad en diferentes ciclos (Tena 1998).

Algunas alternativas que han sido ampliamente recomendadas para inhibir el crecimiento de arvenses, son los cultivos de cobertura con especies leguminosas, las cuales se introducen en las rotaciones de cultivos para proporcionar servicios benéficos al agroecosistema (Terry et al. 1996, Teasdale 2005).

Los cultivos de cobertura fueron parte integral en la mayoría de los sistemas agrícolas de los países desarrollados hasta los años cincuenta, hasta esa época las plantas de cobertura y los desechos animales fueron claves para manejar la fertilidad del suelo y sostener la productividad de granos; conforme la agricultura se hizo más especializada, se introdujo la aplicación de fertilizantes como mecanismo para proveer una fuente de $\mathrm{N}$ fácilmente disponible y el empleo de plantas de cobertura fue decayendo rápidamente en esos países (Sanchol y Cervantes 1997).

Actualmente la práctica de cultivos de cobertura con A. pintoi en cultivos perennes como banano, se ha utilizado en Suramérica por presentar gran velocidad de descomposición, crecimiento rastrero, y soportar diferentes intensidades de sombra y desplazar especies arvenses (Pérez 1997, Vargas 1997, Espindola et al. 2006a, Espindola et al. 2006b). No obstante, no existen estudios para plantaciones de plátano macho en México; por tal razón, el objetivo de este trabajo fue determinar el efecto de A. pintoi en el control de arvenses en plantaciones de plátano macho.

\section{MATERIALES Y MÉTODOS}

\section{Localización del experimento}

El experimento fue establecido en la Ranchería Habanero segunda sección del Municipio de Cárdenas, Tabasco, en el sureste de México; y se evaluó entre octubre del 2008 hasta agosto del 2009. El clima es cálido húmedo Am (f)w”(i'), con lluvias en verano, un 
porcentaje de precipitación invernal mayor a 10,2 y la precipitación del mes más seco menor de $60 \mathrm{~mm}$. Una época seca marcada de marzo a mayo y un periodo seco corto de julio-agosto, localmente conocida como canícula. La variación térmica anual es entre $5^{\circ} \mathrm{C}$ y $7^{\circ} \mathrm{C}$ (García 1973). La temperatura anual promedio mínima es de $23,1^{\circ} \mathrm{C}$ y máxima es de $29^{\circ} \mathrm{C}$ (INEGI 2005). Se encuentra a una altitud de $11 \mathrm{msnm}$ con precipitación promedio de $1944 \mathrm{~mm}$, (INEGI 2005). Los suelos del área son fluvisoles eutricos (Palma-López et al. 2007), localizada en las coordenadas UTM 468679 UTH 199064.

Los resultados del análisis físico-químico del suelo $(0-15 \mathrm{~cm})$ al inicio del experimento presentaron los siguientes valores: $\mathrm{pH}$ en $\mathrm{H}_{2} \mathrm{O}, 5,8$; materia orgánica, 2,34\%; $\mathrm{N}$ total, 0,1\%; P Olsen, 28,2 mg/kg; $\mathrm{Ca}^{2+}, 28,2$ $\mathrm{cmol} / \mathrm{kg} ; \mathrm{Mg}^{2+}, 9,27 \mathrm{cmol} / \mathrm{kg} ; \mathrm{K}^{+}, 1,68 \mathrm{cmol} / \mathrm{kg}$. De esta manera se permite inferir que las características físico-químicas del suelo son adecuadas para el establecimiento de A pintoi.

El experimento se estableció en octubre del 2008 en dos plantaciones establecidas con plátano macho con edad de 3,5 años y una densidad de 144 plantas $/ 420 \mathrm{~m}^{2}$, con un nivel de sombra en estrato herbáceo de $45 \%$ ( $s= \pm 11,1)$ y $50 \%$ ( $s= \pm 13,2)$, la cual está en función de la distancia de siembra $(2 \times 2 \mathrm{~m})$, en la región el patrón de siembra es el mismo en plátano macho.

El material vegetal que cubría el suelo antes de establecer el experimento se eliminó con machete; esta vegetación estaba constituida principalmente por Priva lappulace (L.) Pers y Sida acuta Burm. Posteriormente se procedió al establecimiento de la cobertura de $A$. pintoi, en forma directa, eliminando la superficie del suelo con la punta de un machete. Se utilizaron estolones de $20-25 \mathrm{~cm}$ de longitud, los cuales se enterraron en el suelo a unos $5 \mathrm{~cm}$ de profundidad, distribuyéndose a una distancia de $30 \times 30 \mathrm{~cm}$ entre las hileras y los espacios vacíos de las plantas de plátano macho. La densidad aproximada de estolones fue de 111 000/ha. El tratamiento con arvenses fue considerado testigo, éste fue establecido dejando toda la vegetación herbácea desde el inicio hasta el final del experimento sin ningún manejo.

El manejo agronómico de las plantas de plátano macho en las parcelas experimentales, consistió en hacer: a) labores mensuales como la eliminación de hojas agobiadas o enfermas y la cosecha de los racimos después de tres meses de desarrollo; b) cada mes y medio, la selección del hijo de producción y; c) limpieza de la base de las plantas de plátano macho (Pérez 1997).

El control de arvenses se llevó a cabo en la sección con A. pintoi mediante la utilización de chapeas manuales (machete), durante los primeros cinco meses después de la siembra de la leguminosa con el fin de proporcionar las condiciones idóneas para el prendimiento de los estolones (Vargas 1997).

\section{Determinación de niveles de sombra}

Para la determinación de los niveles de sombra, se realizaron mediciones de radiación fotosintéticamente activa $\left(\mu \mathrm{mol} \mathrm{m}^{-2} \mathrm{~s}^{-1}\right)$ bajo dosel y a campo abierto a un metro del suelo con un sensor quantum Li-190SA, conectado a un datalogger 1400. Se tomaron 100 lecturas, realizando el recorrido en forma de zig-zag, de modo de integrar todas las situaciones de radiación bajo dosel, en cada sitio se calculó la media general para determinar la radiación fotosintéticamente activa (RAFA), de la misma manera se realizaron mediciones fuera de las parcelas para calcular esta a cielo abierto (Ica). Con base en las mediciones de RAFA se estimó la radiación disponible para el crecimiento del A. pintoi y arvenses. Según Bellow y Nair (2003) fue definida como la diferencia de fracción de RAFA respecto a la condición de RAFA a cielo abierto (ecuación 1).

$$
\text { Sombra }=[1-(\mathrm{Ibd} / \mathrm{Ica})]^{*} 100
$$

Donde: Sombra $=$ nivel de sombra (\%); Ibd: radiación fotosintéticamente activa bajo dosel de plátano; Ica: radiación fotosintéticamente activa a cielo abierto.

\section{Diseño experimental}

Se utilizó un diseño de bloques completos al azar, con arreglo factorial (dos tipos de sombreado $\mathrm{X}$ dos tipos de cobertura vegetal) con tres repeticiones (Cuadro 1). Se realizó el análisis de varianza del experimento factorial y la comparación múltiple de medias (Tukey $\mathrm{p}=0,05)$ para las variables: índice de Shannon-Wiever $\left(H^{\prime}\right)$, MS de arvenses y A. pintoi, con el paquete estadístico SAS (SAS. Institute, Inc. 2002). 
Cuadro 1. Descripción de los tratamientos para el control de arvenses en una plantación de plátano macho. Cárdenas, Tabasco, México. 2008.

\begin{tabular}{cc}
\hline Nivel de sombra $(\%)$ & Tratamiento \\
\hline $45 \pm 11,1$ & Suelo con arvenses \\
$45 \pm 11,1$ & Suelo con $A$. pintoi \\
$50 \pm 13,2$ & Suelo con arvenses \\
$50 \pm 13,2$ & Suelo con $A$. pintoi \\
\hline
\end{tabular}

\section{Muestreo de arvenses}

Los muestreos de las poblaciones de arvenses se realizaron en septiembre de 2008 (antes de la siembra del A. pintoi) y agosto de 2009 (al finalizar el estudio); el procedimiento consistió en trazar una línea Canfield $(0,10 \times 10 \mathrm{~m})$ al azar en cada sitio. Del área dentro de la línea Canfield se midió: altura, dominancia, valores absolutos y relativos e índice de importancia para cada especie presente. Se extrajeron las arvenses para identificación taxonómica de familia, género y especie empleando claves botánicas. Para el mes de agosto del 2009 se trazó una línea Canfield en todas las repeticiones de cada tratamiento y se repitió la evaluación de variables para comparar la presencia de las arvenses o aparición de otras.

La dominancia, valores absolutos y relativos de las especies vegetales en el área de estudio se determinó aplicando las siguientes fórmulas (Caamal 2004):

Dominancia relativa (Dr)

$$
\operatorname{Dr}=(\mathrm{Ae} / \mathrm{At}) * 100
$$

Donde: $\mathrm{Ae}=$ Área basal de cada especie; At $=$ Área basal del total de especies.

Abundancia (A)

$A=(N / T) * 100$

Donde: $\mathrm{N}=$ Número de individuos de cada especie $\mathrm{T}=$ Total de individuos.

Frecuencia Absoluta (F):

$\mathrm{F}=(\mathrm{Po} / \mathrm{Npo}) * 100$
Donde: Po = Número de puntos de ocurrencia de la especie; $\mathrm{Npo}=$ Número total de puntos.

Frecuencia relativa $(\mathrm{Fr})$ :

$\mathrm{Fr}=\left(\mathrm{F} / \sum \mathrm{F}\right)^{*} 100$

Donde: $\mathrm{F}=$ Frecuencia absoluta; $\Sigma \mathrm{F}=$ Sumatoria de las frecuencias de todas las especies.

La importancia de las especies vegetales en el área de estudio se determinó mediante el valor de importancia (VI) (Caamal 2004):

$$
\mathrm{VI}(\%)=\mathrm{A}+\mathrm{Fr}+\mathrm{Dr}
$$

Donde: $\mathrm{A}=$ Densidad relativa; $\mathrm{Fr}=$ Frecuencia relativa; $\mathrm{Dr}=$ Dominancia relativa.

\section{Riqueza de especies}

\section{Índice de diversidad de Shannon-Wiever $\left(H^{\prime}\right)$}

El índice de Shannon-Weaver es un índice que mientras más alto es el valor, mayor es la diversidad y la comunidad está menos dominada por una o pocas especies. Los valores mayores a 3,5 indican rareza y abundancia de las especies más equitativas (Odum 1978). Este índice se calculó con la siguiente ecuación:

$$
\mathrm{H}^{\prime}=-\Sigma(\mathrm{ni} / \mathrm{n}) \ln (\mathrm{ni} / \mathrm{n})
$$

Donde ni $=$ Valor de importancia para cada especie $; n=$ Total de valores de importancia; $\ln =$ logaritmo natural.

\section{Índice de similitud de Sörensen (S)}

La semejanza florística entre los dos niveles de sombra se determinó con la siguiente ecuación:

$$
\text { Sörensen }(S)=2 C /(A+B)
$$

Donde $\mathrm{A}=$ Número de especies presentes con 45\% ( $\mathrm{s}= \pm 11,1)$ sombra; $\mathrm{B}=$ Número de especies presentes con $50 \%(s= \pm 13,2)$ sombra; $C=$ Número de las especies comunes en ambos niveles de sombra. 
Valores iguales o cercanos a 1 indican una mayor semejanza florística (Odum 1978).

\section{Biomasa aérea (BA) en las arvenses y A. pintoi}

Para estimar la producción de BA de las arvenses y A. pintoi se realizó un muestreo al término del trabajo con un cuadro de madera $0,50 \times 0,50 \mathrm{~m}(0,25$ $\mathrm{m}^{2}$ ), con cinco puntos de muestreo por unidad experimental en forma de zig-zag, cosechando con tijeras a ras del suelo toda la vegetación herbácea (A. pintoi y arvenses) dentro del cuadro. En los tratamientos con A. pintoi se realizó la separación entre especies arvenses y esta leguminosa. La BA se pesó en fresco y se colocó en bolsas de papel para secar en estufa durante $72 \mathrm{~h}$ o hasta peso constante a $70^{\circ} \mathrm{C}$. Con los datos de la materia seca (MS), se calculó el promedio de la BA de arvenses y A. pintoi; posteriormente estas variables se extrapolaron a $\mathrm{kg} \mathrm{MS} / \mathrm{ha} /$ tratamiento.

\section{Efectividad en el control de arvenses}

Para obtener el efecto supresor de la cobertura de A. pintoi en el control de arvenses, se determinó su porcentaje de efectividad con la ecuación 5 (Sanchol y Cervantes 1997) en cada nivel de sombra. Se utilizó el peso seco de la BA producida en cada tratamiento con arvenses, así como la producida con A. pintoi. Efectividad en el control de arvenses definido como:

$$
\text { Efectividad }=\left[\left(\mathrm{A}_{\mathrm{t}}-\mathrm{A}_{\mathrm{A}}\right) / \mathrm{A}_{\mathrm{t}}\right]^{*} 100
$$

Donde: $\mathrm{A}_{\mathrm{t}}=\mathrm{MS}$ de tratamiento con arvenses; $\mathrm{A}_{\mathrm{A}}$ $=$ MS de tratamiento con A. pintoi.

\section{RESULTADOS Y DISCUSIÓN}

Flora arvense inicial en plátano macho con 45 ( $s=$ $\pm 11,1)$ y $50 \%$ ( $(s= \pm 13,2)$ de sombra en estrato herbáceo al inicio del experimento

En el tratamiento con $45 \%(s= \pm 11,1)$ de sombra en su estrato inferior, se identificaron 15 especies de arvenses pertenecientes a siete familias y con $50 \%$ $(s= \pm 13,2)$ de sombra cinco especies pertenecientes a cinco de éstas. En el tratamiento con 50\% ( $\mathrm{s}= \pm 13,2)$ de sombra en estrato herbáceo, el mayor número de familias (5) pertenecen al tipo de hoja ancha, esto es porque se trata de una plantación establecida con mayor sombra y humedad que genera un sitio propicio para el desarrollo de estas especies (Cuadro 2) (Pinilla y

Cuadro 2. Arvenses encontradas en los dos niveles de sombra que generó en una plantación de plátano macho. Cárdenas, Tabasco, México. 2008.

\begin{tabular}{llcl}
\hline Familia & Especies & Sitio & Clase \\
\hline Araceae & Syngoniun podophyllum Schott & I, II & Monocotiledónea \\
Capparaceae & Cleome serrata Jacq. & II & Dicotiledónea \\
Commelinaceae & Commelina diffusa N. L. Burm. & I & Monocotiledónea \\
Asteraceae & B. pilosa L. & I & Dicotiledónea \\
Euphorbiaceae & Acalypha alopecuroides Jacq. & I & Dicotiledónea \\
& A. virginica L. & I & Dicotiledónea \\
& Caperonia palustris (L.) St. Hil. & I & Dicotiledónea \\
& Euphorbia heterophylla L. & I & Dicotiledónea \\
& E. hirta L. & I & Dicotiledónea \\
Malvaceae & Heliocarpus Donnell-Smithii Rose & I & Dicotiledónea \\
Poaceae & S. acuta Burm f. Huinar & I, II & Dicotiledónea \\
& E. colonum (L.) Link & I & Monocotiledónea \\
Verbenaceae & Leptochloa filiformis (Lam.) Beauv. & I, II & Monocotiledónea \\
& Lantana camara L. var parviflora Mold. & I & Dicotiledónea \\
& P. lappulaceae (L.) Pers & I, II & Dicotiledónea \\
\hline
\end{tabular}

$\mathrm{I}=45 \%(\mathrm{~s}= \pm 11,1)$ sombra en estrato herbáceo.

$\mathrm{II}=50 \%(\mathrm{~s}= \pm 13,2)$ Sombra en estrato herbáceo. 
García 2002); presentando mayor número de especies (5) la familia Euphorbiaceae. Como menciona van Rijn (2000), la composición de flora arvense es diversa de región a región debido a las diferentes condiciones de topografía, clima, suelo y diferentes actividades del hombre, pero ciertas especies son dominantes en muchas regiones como son las Asteraceae (Bidens pilosa L.), Euphorbiaceae (Euphobia sp.), Poaceae (Echinochloa colonum [L.] Link, Digitaria sanguinalis [L.] Scop.).

Al inicio del trabajo, el índice de Shannon-Wiever $\left(H^{\prime}\right)$, señala que el estrato herbáceo con $45 \%(\mathrm{~s}= \pm$ $11,1)$ de sombra $\left(H^{\prime}=2,4\right)$ posee una mayor diversidad con respecto al estrato herbáceo con $50 \%$ ( $\mathrm{s}= \pm$ $13,2)$ de sombra $\left(H^{\prime}=1,3\right)$ y el índice de similitud de Sörensen $(S)$ para los dos niveles de sombra fue bajo $(0,3)$, debido a que en la plantación con $50 \%$ ( $\mathrm{s}= \pm$ $13,2)$ sombra se encontró una dominancia de $P$. lappulaceae (Cuadro 3). La baja similitud y la diferencia en los índices de Shannon-Wiever entre los sitios es una evidencia a favor que el uso de los diferentes herbicidas por parte de diversos productores afecta algunas especies e influye en la proliferación de una u otras especies (Labrada 2000) así como el intervalo de tiempo para control de arvenses.

Cuadro 3. Índice de Shannon-Wiever y Sörensen entre las especies de arvenses presentes para cada tratamiento a niveles de sombra en plantacion de plátano macho al inicio del trabajo, en Cárdenas, Tabasco, México. 2008.

\begin{tabular}{ccc}
\hline Nivel de sombra $(\%)$ & Índice & Valor \\
\hline $45 \pm 11,1$ & Shannon-Wiever & 2,4 \\
$50 \pm 13,2$ & Shannon-Wiever & 1,3 \\
$45-50$ & Sörensen & 0,3 \\
\hline
\end{tabular}

Valor de importancia (VI) de arvenses al inicio del trabajo

En las Figuras 1 y 2, se presentan los valores de importancia para las especies presentes en los dos niveles de sombra, al inicio del trabajo. Se observó que las especies con valores de importancia más altos, a un $45 \%(s= \pm 11,1)$ de sombra en el estrato herbáceo, en orden decreciente fueron: S. acuta Burm

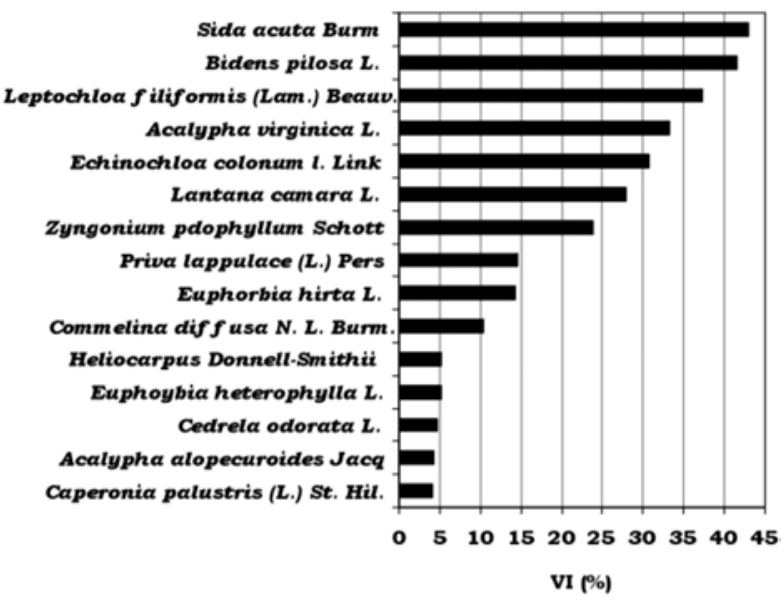

Figura 1. Valor de importancia de las arvenses para un nivel de sombra $45 \%(s= \pm 11,1)$ en estrato herbáceo. Cárdenas, Tabasco, México. 2008.

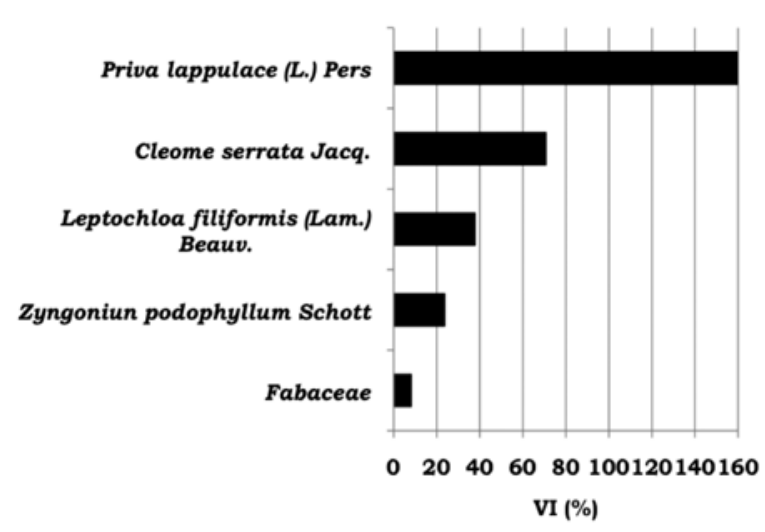

Figura 2. Valor de importancia de las arvenses para un nivel de sombra $50 \%(s= \pm 13,2)$ en estrato herbáceo. Cárdenas, Tabasco, México. 2008.

f. Huinar, B.B. pilosa L., L. filiformis (Lam.) Beauv., A. virginica L., E. colonum (L.) Link, L. camara L. var parviflora Mold., S. podophyllum Schott, siendo las especies que mayor cobertura tienen en el área. van Rijn (2000) cita que las semillas de S. acuta Burm f. Huinar tienen una longevidad de seis años en un suelo tropical, la cual al presentar las condiciones propicias para su germinación provoca aumentos en la población de arvenses. Rzedowskii y Rzedowskii (1979) mencionan que B. pilosa L., es una especie de origen local; su antigüedad es suficiente para no 
dejar desplazarse por arvenses eurasiáticas. Labrada (2000), cita que E. colonum (L.) Link y Cynodon dactylon (L.) Pers. son dos de las principales especies de arvenses asociadas a plantaciones de banano y plátano, donde la práctica del corte no evita totalmente la competencia de C. dactylon (L.) Pers. con el cultivo sino que la estimula. Por otro lado, según Agüero et al. (2008) las aplicaciones constantes de paraquat, favorecen el dominio de monocotiledóneas en estas plantaciones y las de glifosato favorecen las de hoja ancha (dicotiledóneas) por ser herbicidas elaborados para controlar un determinado grupo de arvenses.

En la plantación de plátano macho con $50 \%$ (s= $\pm 13,2$ ) de sombra en estrato herbáceo solo se encontraron cinco especies de las cuales P. lappulaceae (L.) Pers es la que tiene un mayor VI (160\%), siendo una especie dominante en el área, por lo cual se produce un índice de diversidad bajo en este sitio (Figura 2). Rzedowskii y Rzedowskii (2002) consideran esta especie como una arvense agresiva y peligrosa sin problemas de supervivencia.

\section{Respuesta de la flora arvense a los once meses des- pués de establecer los tratamientos}

A los once meses de establecidos los tratamientos se realizaron muestreos de arvenses, para comparar las especies encontradas por tratamiento en cada nivel de sombra. Los valores de $H^{\prime}$ no presentaron diferencias significativas (Tukey, $p=0,05$ ) (Cuadro 4). El valor de

Cuadro 4. Índice de Shannon-Wiever entre las especies presentes para cada tratamiento a dos niveles de sombra en plantacion de plátano macho, a los 11 meses de establecimiento del experimento. Cárdenas, Tabasco, México. 2009.

\begin{tabular}{clc}
\hline $\begin{array}{c}\text { Nivel de } \\
\text { Sombra } \\
(\%)\end{array}$ & \multicolumn{1}{c}{ Tratamiento } & $\begin{array}{c}\text { Valor de } \mathbf{H}^{\prime} \text { (ín- } \\
\text { dice de Shan- } \\
\text { non-Wiever) }\end{array}$ \\
\hline $45 \pm 11,1$ & Suelo con Arvenses & $1,3 \pm 0,6 \mathrm{a}^{*}$ \\
$45 \pm 11,1$ & Suelo con A. pintoi & $1,6 \pm 0,2 \mathrm{a}$ \\
$50 \pm 13,2$ & Suelo con Arvenses & $1,2 \pm 0,4 \mathrm{a}$ \\
$50 \pm 13,2$ & Suelo con A. pintoi & $1,5 \pm 0,6 \mathrm{a}$ \\
$45-50$ & Sörensen & 0,8 \\
\hline
\end{tabular}

*Medias con la misma letra no presentan diferencia significativa al nivel del 5\% de probabilidad según la prueba de Tukey.
$H^{\prime}$ en el nivel de sombra $45 \%$ ( $\left.= \pm 11,1\right)$ disminuyó en un $40 \%$ con respecto al obtenido antes del establecimiento de los tratamientos, debido a un corte de arvenses con machete, que se llevó a cabo para uniformizar los tratamientos con arvenses y realizar una limpieza para el establecimiento de tratamientos con A. pintoi, favoreciendo así la germinación de especies arvenses con mayor presencia de semillas en el suelo y las especies de crecimiento rastrero que pueden estimular su crecimiento. El índice de similitud de Sörensen entre los niveles de sombra fue de 0,8 , aumentando en un $250 \%$ con respecto al calculado al inicio del estudio.

El cambio en la diversidad espacial y temporal de las comunidades arvenses está influenciado por labores agrícolas; además responde en forma semejante a la vegetación herbácea en condiciones naturales y en predecibles (Perdomo et al. 2004). Así, el banco de semillas en el suelo es bien estimulado, ya que algunas especies necesitan cierta intensidad de luz para su germinación, y ésta es promovida cuando el fruto es cosechado, resultando en un aumento en la penetración de luz hasta el suelo lo que provoca la emergencia de arvenses (van Rijn 2000).

Hay evidencias que la diferencia de sombra del $5 \%$ entre los dos sitios, tuvo influencia en la comunidad de arvenses, ésta entre ambos sitios es resultado, como indica Pons (1992), de la respuesta germinativa entre especies de arvenses a las variaciones en la cantidad de luz.

En los tratamientos con A. pintoi se encontró que esta especie alcanza el mayor VI sobre las especies arvenses; entre las presentes en esta asociación con un VI mayor de $50 \%(\mathrm{~s}= \pm 13,2)$ se encuentran: $P$. paniculatum L., E. hirta L., P. lappulaceae (L.) Pers. En el tratamiento con solo arvenses se encontró que Talinum triangulare (Jacq.) Willd alcanza el mayor VI $(253,8 \%)$ considerándose una especie dominante en el cultivo y de difícil control químico y manual, junto con $S$. podophyllum Schott; las especies: P. paniculatum L.; $C$. diffusa N. L. Burm y P. lappulaceae (L.) Pers tuvieron un VI mayor a 50\% ( $\mathrm{s}= \pm 13,2)$ (Figura 3).

El A. pintoi al nivel 50\% (s= $= \pm 13,2)$ de sombra fue el más dominante (VI promedio de 107\%) sobre las arvenses: T. triangulare (Jacq.) Willd y P. paniculatum L. (Figura 4); en estos tratamientos las especies arvenses son las que se encontraban con muy pocos individuos antes del deshierbe para la siembra del $A$. pintoi y que se desarrollaron a la par de éste. En los tratamientos con arvenses la especie con VI mayor 


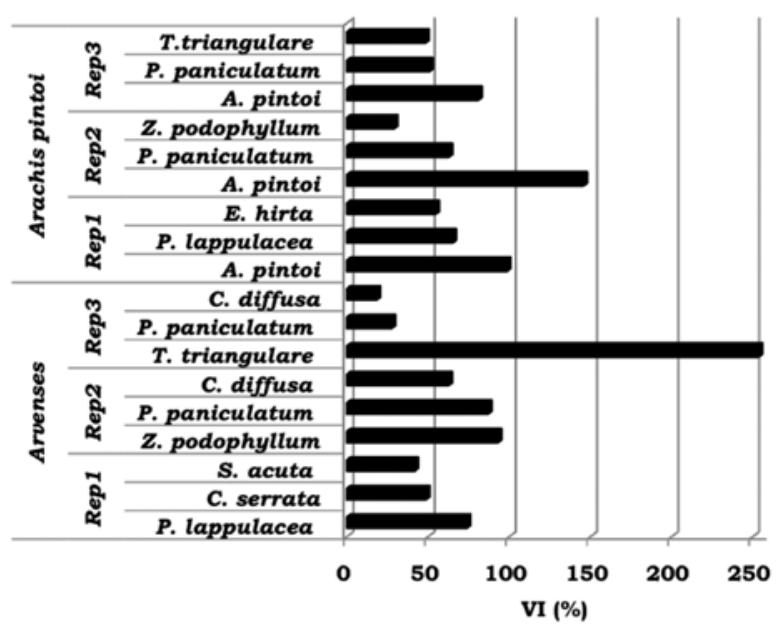

Figura 3. Especies de arvenses con valor de importancia (VI)* más alto para cada tratamiento/repetición a nivel $45 \%$ ( $s= \pm 11,1)$ de sombra. Cárdenas, Tabasco, México. 2009.

* VI $(\%)=\mathrm{A}+\mathrm{Fr}+\mathrm{Dr}(\mathrm{A}=$ Densidad relativa $; \mathrm{Fr}=$ Frecuencia relativa; $\mathrm{Dr}=$ Dominancia relativa).

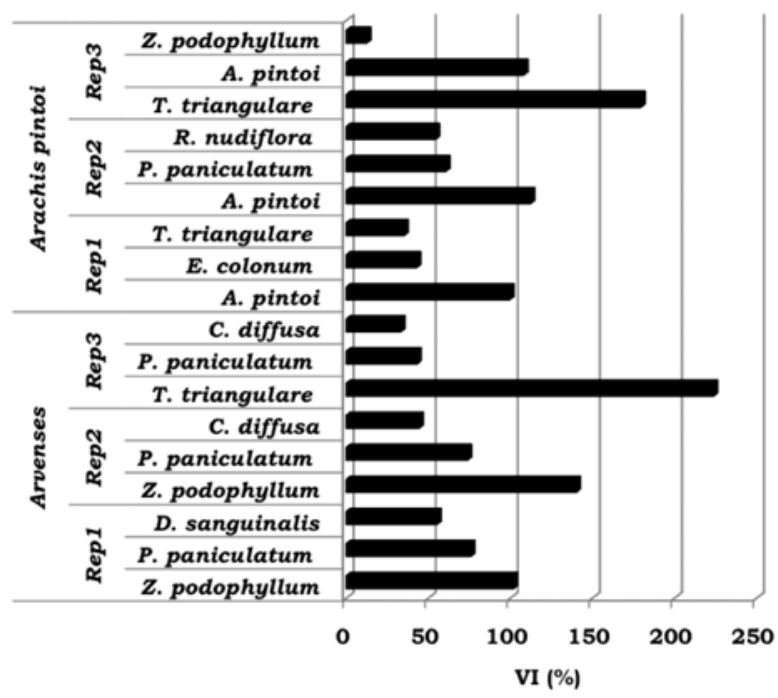

Figura 4. Especies de arvenses con valor de importancia (VI)* más alto para cada tratamiento/repetición a nivel $50 \%(s= \pm 13,2)$ de sombra. Cárdenas, Tabasco, México. 2009.

$* \mathrm{VI}(\%)=\mathrm{A}+\mathrm{Fr}+\operatorname{Dr}(\mathrm{A}=$ Densidad relativa $; \mathrm{Fr}=$ Frecuencia relativa; $\mathrm{Dr}=$ Dominancia relativa $)$. a $200 \%$ es $T$. triangulare (Jacq.) Willd seguida de $S$. podophyllum Schott y $P$. paniculatum $\mathrm{L}$.

Las especies $T$. triangulare (Jacq.) Willd y $S$. podophyllum Schott. presentaron mayor dominancia en el área de estudio y los agricultores las han identificado como plantas tolerantes a los herbicidas paraquat y glifosato. Del mismo modo se tiene problemas al deshierbe, reproducción sexual y asexual, y esta práctica promueve la dispersión y aumento de las poblaciones dentro de la plantación, siendo determinante el control en la dinámica de las arvenses en el agroecosistema. Como indica van Rijn (2000), T. triangulare (Jacq.) Willd es una especie perenne que se desarrolla muy bien tanto en condiciones de sombra como a luz directa. Aguiero et al. (2008) menciona que $S$. podophyllum Schott es poco susceptible a los métodos de control (glifosato, paraquat y deshierbe manual) en algunas zonas bananeras de Costa Rica, y por su habilidad para competir tiende a dominar este agroecosistema; algunas características que presenta esta especie son un mecanismo llamado escototropismo, mediante el cual las plántulas crecen en dirección a la sombra y cuando alcanzan este objetivo posteriormente trepan sobre el pseudotallo del banano, siguiendo los gradientes de luz, causando interferencia a la hoja candela, impidiendo la emisión del racimo, o dificultando la cosecha cuando envuelve el racimo.

\section{Materia seca (MS) de arvenses y A. pintoi en cultivo de plátano macho}

\section{Materia seca (MS) de arvenses}

De acuerdo con prueba Tukey $(p=0,05)$ en el sitio con $55 \%$ de luz no se encontraron diferencias estadísticamente significativas en la producción de MS entre los tratamientos; contrario al sitio con 50\% $(s= \pm 13,2)$ de luz, donde se encontraron diferencias estadísticas, siendo un 30\% mayor la producción de MS por arvenses a la MS producida en los tratamientos con A. pintoi (Cuadro 5). La mayor producción de MS fue en el tratamiento de suelo con arvenses a 50\% $(\mathrm{s}= \pm 13,2)$ sombra $\left(375,37 \pm 83,39 \mathrm{~g} / \mathrm{m}^{2}\right)$ respondiendo a la dominancia de las especies $T$. triangulare y $S$. podophyllum, las cuales alcanzaron esta dominancia probablemente por la gran humedad que se presentó en los meses de junio y julio, buena adaptación a la sombra, y selección por aplicación de herbicidas desde 
Cuadro 5. Materia seca de arvenses y A. pintoi producida en cada tratamiento a dos niveles de sombra en cultivo de plátano macho. Cárdenas, Tabasco, México. 2009.

\begin{tabular}{clc}
\hline $\begin{array}{c}\text { Nivel de } \\
\text { sombra }(\%)\end{array}$ & Tratamiento & $\begin{array}{c}\text { MS } \\
\left(\mathbf{g} / \mathbf{m}^{2}\right)\end{array}$ \\
\hline $45 \pm 11,1$ & Suelo con arvenses & $266,9 \pm 91,7 \mathrm{~b} *$ \\
$45 \pm 11,1$ & Suelo con A.pintoi & $326,1 \pm 78,1 \mathrm{ab}$ \\
$50 \pm 13,2$ & Suelo con arvenses & $375,3 \pm 83,4 \mathrm{a}$ \\
$50 \pm 13,2$ & Suelo con A. pintoi & $261 \pm 83,4 \mathrm{~b}$ \\
\hline
\end{tabular}

* Medias con la misma letra no presentan diferencia significativa al nivel del 5\% de probabilidad según la prueba de Tukey.

el establecimiento de la plantación. La producción de MS promedio de arvenses en un nivel de 50\% ( $\mathrm{s}= \pm$ 13,2 ) de sombra fue $10 \%$ mayor a la MS reportada por Espindola et al. (2006a) en temporada de secas y 50\% $(s= \pm 13,2)$ menor para la temporada de lluvias, para las arvenses con una dominancia de Panicum maximum en cultivo de banano en Brasil.

\section{Materia seca (MS) producida por A. pintoi}

La MS producida A. pintoi promedio fue obtenida al separarla de la MS de arvenses. Se obtuvo una MS de A. pintoi promedio a los once meses después de la siembra en $45 \%$ de sombra de $198,9 \mathrm{~g} / \mathrm{m}^{2}$, mayor a la producida con $50 \%$ de sombra $\left(150,4 \mathrm{~g} / \mathrm{m}^{2}\right)$. De acuerdo a la prueba de Tukey $(p=0,05)$ no se encontraron diferencias estadísticas significativas para ambos niveles de sombra (Cuadro 6).

Cuadro 6. Materia seca de A. pintoi producida en cada nivel de sombra en plantación de plátano macho. Cárdenas, Tabasco. México. 2009.

\begin{tabular}{ccc}
\hline $\begin{array}{c}\text { Nivel de } \\
\text { sombra (\%) }\end{array}$ & Tratamiento & $\begin{array}{c}\text { MS } \\
\left(\mathbf{g} / \mathbf{m}^{\mathbf{2}}\right)\end{array}$ \\
\hline $45 \pm 11,1$ & Suelo con A. pintoi & $198,9 \pm 79 \mathrm{a}^{*}$ \\
$50 \pm 13,2$ & Suelo con A. pintoi & $150,4 \pm 77 \mathrm{a}$ \\
\hline
\end{tabular}

* Medias con la misma letra no presentan diferencia significativa al nivel del $5 \%$ de probabilidad según la prueba de Tukey.
Al extrapolar la MS producida por A. pintoi a toneladas por hectárea, se obtuvo un valor de 1,98 t/ha en $45 \%(\mathrm{~s}= \pm 11,1)$ de sombra y $1,5 \mathrm{t} / \mathrm{ha}$ con $50 \%$ ( $\mathrm{s}= \pm$ 13,2 ) de sombra. Estos resultados se encuentran dentro de los intervalos encontrados por algunos autores que han evaluado el establecimiento de esta leguminosa dentro de plantaciones perennes como los cítricos. Para este frutal, Rincón (1999), después de nueve meses de establecimiento, reporta un promedio de 0,67 t/ha para A. pintoi CIAT 18744 y 18748; y Dalcolmo et al. (1999), después de 10 meses de siembra, una producción de MS de 3,32 t/ha en plantaciones de cítricos. En plantaciones de banano Espindola et al. (2006a) indicaron una MS en temporada de seca de 3,4 t/ha y en temporada de lluvias 4,2 t/ha; y Espindola et al. (2000), Perin et al. (2003) mostraron una acumulación de $12 \mathrm{t} /$ ha en 22 meses después del establecimiento.

Existen diversos factores que influyen directamente en la producción de MS de A. pintoi en América tropical, por la diferencia en prácticas agrícolas y ambientales existentes en el continente, como lo han reportado algunos autores (Argel y Villarreal 1998, Rincón 1999, Valentim et al. 2001, Nascimento 2006) han evaluado esta leguminosa.

En condiciones de $A$. pintoi en monocultivo a pleno sol, Machado et al. (2005) encontraron producciones más altas de MS (7,1 t/ha) en poblaciones más densas, a distancias menores entre líneas y entre plantas (espaciamiento de 0,25 X 0,25 m); y Puertas et al. (2008), a los doce meses después del establecimiento, obtuvieron una producción de 5,07 t MS/ha con un espaciamiento de $0,50 \times 0,50 \mathrm{~m}$.

A. pintoi con $50 \%(s= \pm 13,2)$ de sombra produce $80 \%$ más de MS a la que se obtienen a pleno sol, lo cual es buena indicación de la plasticidad de esta especie por la tolerancia a la sombra (Zelada e Ibrahim 1995). Asimismo, Fisher y Cruz (1995) notaron que la producción de MS de A. pintoi tendía a aumentar cuando el nivel de sombra fue incrementado.

\section{Efectividad en el control de arvenses de Arachis pintoi en cultivo de plátano macho}

$\mathrm{Al}$ aplicar la ecuación 9 para estimar la efectividad en el control de arvenses (Sanchol y Cervantes, 1997), se encontró que en los dos niveles de sombra A. pintoi redujo la presencia de arvenses en el cultivo de plátano macho con una efectividad del 52,34\% (45 
$\pm 11,1 \%$ de sombra) y $70,50 \%(50 \pm 13,2 \%$ de sombra) encontrándose especies arvenses con capacidad reproductiva asexual de difícil control. La efectividad en el control de arvenses por A. pintoi fue $20 \%$ mayor con un nivel de sombra de $50 \%(\mathrm{~s}= \pm 13,2)$ que en el nivel de $45 \%(s= \pm 11,1)$ de sombra (Figura 5). Al interferir el $A$. pintoi en la germinación de semillas arvenses, provocó una disminución en sus poblaciones, lo que demuestra la capacidad de esta leguminosa para controlar arvenses en las plantaciones de plátano macho de similares condiciones edafoclimáticas; por esta capacidad, y por eliminar el control mecánico de arvenses, el uso de $A$. pintoi ha sido recomendado por la Federación Nacional de Cafeteros de Colombia en las plantaciones de café (Pérez y Pizarro 2005). Estos resultados se asemejan a los encontrados para otras leguminosas por Ortiz (1995) a nivel invernadero, encontró que Aeschynomene americana L. y Sesbania emerus (Aubl.) Urb. lograron disminuir en un $80 \%$ las arvenses: E. colona, L. filiformis (L.) Beauv. y C. diffusa Burm F.; Gutiérrez et al. (2002), en el cultivo del plátano, encontraron que Centrosema plumieri (Turp. ex Pers.) Benth, Teramnus labialis (L. F.) Spreng y Stylosanthes guianensis (Aubl.) Sw. a dos años de establecimiento obtuvieron una reducción significativa de especies arvenses.

Con el establecimiento a densidades altas de $A$. pintoi se logra un porcentaje de cobertura en el menor tiempo posible, lo cual es ocasionado probablemente por la disminución del porcentaje de germinación de semillas de arvenses presentes en el agroecosistema de

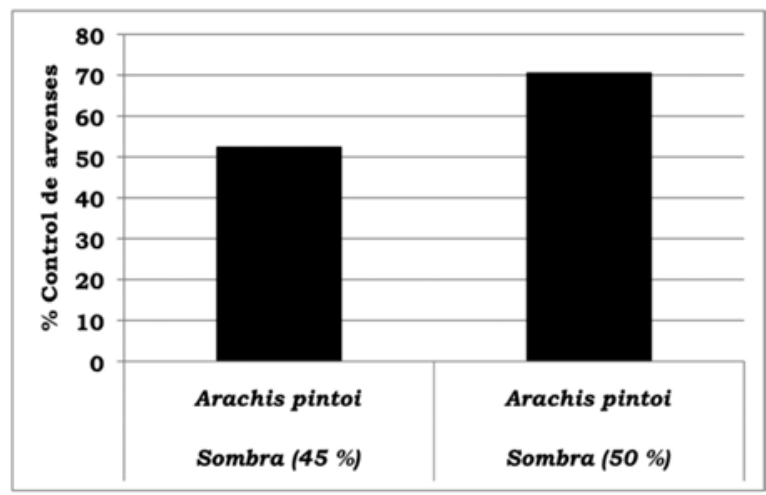

Figura 5. Efectividad en control de arvenses con A. pintoi en dos niveles de sombra en plantación de plátano macho. Cárdenas, Tabasco, México. 2009. plátano macho (Isaac et al. 2007), con una eficiencia en el control de arvenses a los dos meses después de su establecimiento, usando A. pintoi (52,1\%), Desmodium heterocarpon (86,7\%), y Mucuna pruriens $(43,3 \%)$ en plantaciones de banano; en este experimento la distancia de siembra para A. pintoi fue $16 \mathrm{~cm}$.

La efectividad promedio de A. pintoi (62\%) encontrada en este trabajo es $20 \%$ menor al compararla con resultados de aplicaciones con herbicidas paraquat y glifosato, en periodo de un año (Gómez 2005). Sin embargo, en este caso conforme se realizan más aplicaciones en el tiempo se van seleccionando arvenses resistentes a estos herbicidas y la efectividad disminuye. Caso contrario el comportamiento de A. pintoi, conforme pasa el tiempo éste va desplazando arvenses y aumenta la efectividad del control por la producción de biomasa.

Después de los once meses de establecido el $A$. pintoi como cobertura viva, redujo las especies arvenses de manera significativa (entre $52 \%$ y $70 \%$ ) en plantaciones de plátano macho. La producción de materia seca encontrada por A. pintoi en niveles de $45 \%$ ( $\mathrm{s}= \pm$ $11,1)$ y $50 \%(\mathrm{~s}= \pm 13,2)$ es semejante a la producida por las arvenses en las condiciones dadas, sin encontrar influencia en el índice de diversidad. Observándose una tendencia positiva en el control de arvenses a través del tiempo de asociación por el aumento en la producción de biomasa de A. pintoi, reduciendo la emergencia de las arvenses porque las semillas que requieren una breve exposición de luz no son inducidas a germinar.

En el agroecosistema plátano macho el amontonamiento de los residuos de cosecha, permiten que la entrada de luz en algunos sectores, y arvenses competitivas bajo condiciones de sombra se vuelvan un problema, ya que se requiere controlarlas aún cuando la plantación haya cerrado, finalmente es importante valorar el efecto de la cosecha, ya que permite que la luz llegue de nuevo al suelo.

\section{LITERATURA CITADA}

Agüero, R; Brenes, S; Rodríguez, AM. 2008. Alternativas para el control químico de Conde (Syngoniun podophyllum Schott) en banano (Musa AAA). Agronomía Mesoamericana 19(2):285-289.

Argel, P; Villareal, M. 1998. Cultivar Porvenir: Nuevo maní forrajero perenne (Arachis pintoi Krap. y Greg. 
nom. nud., CIAT 18744) Leguminosa herbácea para alimentación animal, el mejoramiento y conservación del suelo y el embellecimiento del paisaje. Ministerio de Agricultura y Ganadería de Costa Rica, Centro Internacional de Agricultura Tropical (CIAT). Boletín técnico $32.32 \mathrm{p}$.

Bellow, J; Nair, P. 2003. Comparing common methods for assessing understory light availability in shaded-perennial agroforestry system. Agricultural and forest meteorology 114:197-211.

Caamal, J. 2004. Arvenses. In Bautista, F; Delfín, H; Palacio, J; Delgado, M. eds. Técnicas de muestreo para manejadores de recursos naturales. UNAM-UAYCONACYT-INE. México. p. 343-362.

Dalcolmo, J; Almeida, D; Guerra, J. G. 1999. Avaliaçã de leguminosas perenes para cobertura de solo em pomar cítrico no municipio de Jerônimo Monteiro, Es. Seropedica: Embrapa Agrobiologia. Boletín Técnico $36.8 \mathrm{p}$.

Espindola J; Rodrigues de Oliveira, S; Carvalho, G; Melo de Souza, C; Perin, A; Guerra, J; Teixeira, M. 2000. Potencial alelopático e controle de plantas invasoras por leguminosas herbáceas perenes consorciadas com bananeira. Seropedica: Embrapa Agrobiologia. Boletín Técnico 47. 8 p.

Espindola, J; Guerra, L; Almeida, D; Texeira, M; Urquiaga, S. 2006a. Descomposicao e liberacao de nutrientes acumulados em leguminosas herbáceas perenes consorciadas com bananeira. R. Bras, Ci. Solo 30(2): 321-328.

Espindola, J; Guerra, L; Almeida, D; Texeira, M; Urquiaga, S; Busquet, R. 2006b. Bananeiras consorciadas com leguminosas herbáceas perenes utilizadas como coberturas vivas. Pesq. Agropec. Bras. 41(3):415-420.

Espinosa, J; Sarukhan, J. 1997. Manual de malezas del valle de México. Instituto de Ecología. Universidad Nacional Autónoma de México. Fondo de Cultura Económica. México, D.F. 407 p.

Fisher, M; Cruz, P. 1995. Algunos aspectos de la ecofisiología de Arachis pintoi. In Kerridge, P; Hardy; B. eds. Biología y agronomía de especies forrajeras de Arachis. Cali, Colombia: CIAT. p. 56-75.

García, E. 1973. Modificaciones al sistema de clasificación climática de Köppen (para adaptarlo a las condiciones de la República Mexicana). Universidad Nacional Autónoma de México. México. 246 p.

Gliessman, S. 2002. Agroecología: procesos ecológicos en agricultura sostenible. Turrialba, Costa Rica. CATIE. 359 p.
Gómez, R. 2005. Efecto del control de malezas con paraquat y glifosato sobre la erosión y pérdida de nutrimentos del suelo en cafeto. Agronomía Mesoamericana 16(1):77-87.

Gutiérrez, I; Pérez, R; Benega, R; Gómez, L. 2002. Coberturas vivas de leguminosas en el plátano (Musa sp.) FHIA 03. Cultivos Tropicales 23(3):11-17.

INEGI (Instituto Nacional de Estadística y Geografía). 2005. Cuaderno Estadístico Municipal Cunduacán, Tabasco. México. 175 p.

Isaac, W; Brathwaite, R; Cohen, J; Bekele, I. 2007. Effects of alternative weed management strategies on Commelina diffusa Burm. Infestations in Fairtrade banana (Musa spp.) in St. Vincent and the Grenadines. Crop Protection 26:1219-1225.

Labrada, R. 2000. Manejo de malezas en bananos y plátanos. In Informe Sobre el taller regional del manejo integrado de plagas en banano y plátano, Venezuela. División de producción y protección vegetal. Organización de las Naciones Unidas para la agricultura y la alimentación. Roma. 61-71 p.

Machado, A; Siewerdt, L; Zonta, E; Vahl, C; Coelho, R; Lauz, O; Affonso, A. 2005. Rendimento do amendoim-forrageiro establecido sob diferentes arranjos populacionais de plantas em planossolo. Ciência Animal Brasileira 6(3):151-162.

Marzocca, A. 1979. Manual de malezas. Ed. Hemisferio Sur. Buenos Aires, Argentina. 564 p.

Muñoz, R. 1985. Contenido de N, P, K, Ca y Mg en arvenses asociadas a 2 genotipos de Maíz (Zea mayz L.) a diferentes fechas de deshierbe en la Chontalpa, Tabasco, México. Tesis de Ingeniería. Colegio Superior de Agricultura tropical.Cárdenas, Tabasco, México. $94 \mathrm{p}$.

Nascimento, I. 2006. O cultivo do amendoin forrageiro. Revista Brasileira de Agrociência, Pelotas 12(4):387-393.

Naylor, R. 2002. Weed management handbook. Blackwell Science-British. Crop Protection Council. Osney Mead, UK. 423 p.

Odum, E. 1978. Ecología: El vínculo entre las ciencias naturales y las sociales. CECSA. México. 295 p.

Ortiz, A. 1995. Aporte de nitrógeno y control de arvenses por el uso de leguminosas en el cultivo del arroz. Tesis. Mag. Sc. Colegio de Postgraduados. Campus Montecillo. México. 116 p.

Palma, D; Cisneros, D; Moreno, C; Rincón-Ramírez, J. 2007. Suelos de Tabasco: su uso y manejo sustentable. Colegio de Postgraduados-ISPROTAB-FUPROTAB. Villahermosa, Tabasco, México. 195 p. 
Palencia, G; Gómez, R; Martín, J. 2006. Manejo sostenible del cultivo del plátano. Corpoica-CORPOBOYACA. Bucaramanga, Colombia. 28 p.

Perdomo, F; Heyke, V; Romero, A; Domínguez, A; Medina, J. 2004. Análisis de SHE, una herramienta para estudiar la diversidad de malezas. Revista Fitotecnia Mexicana 27 (Número especial 1):57-61.

Pérez, N; Pizarro, E. 2005. Potencial forrajero del género Arachis en el trópico americano. IX Seminario de Pastos y forrajes. Brasil. (en línea). Consultado 4 jun. 2009. Disponible en http://www.avpa.ula.ve/ix_seminario_ pastosyforraje/Conferencias/C2-NaylosBastiani.pdf.

Pérez, L. 1997. Evaluación de introducciones de Arachis pintoi como plantas de cobertura viva en banano (Musa AAA), cv. "Gran Enano". CORBANA 22(48): 77-88.

Perin, A; Guerra, J; Teixeira, M. 2003. Cobertura do solo e acumulação de nutrientes pelo amendoim forrageiro. Pesq. Agropec. Bras., Brasilia. 38 (7): 791-796.

Pinilla, C; García, J. 2002. Manejo integrado de arvenses en plantaciones de banano (Musa AAA). In Memorias de la XV Reunión Acorbat. Cartagena de Indias, Colombia. Asociación de Bananeros de Colombia, AUGURA. p. 222-235.

Pons, T. 1992. Seed responses to light. In Fenner, M. ed. Seeds the ecology of regeneration in plant communities. CABI Publishing. London, UK. p. 237-260.

Puertas, F; Arévalo, E; Zuñiga, L; Loli, O; Soplin, H; Baligar V. 2008. Establecimiento de cultivos de cobertura y extracción total de nutrientes en un suelo de trópico húmedo en la amazonía Peruana. Ecología Aplicada 7(1,2):23-28.

Rincón, A. 1999. Maní forrajero (Arachis pintoi), la leguminosa para sistemas sostenibles de producción agropecuaria. Boletín técnico. No. 24. Corporación Colombiana de Investigación Agropecuaria (Corpoica)-Programa Nacional de Transferencia de Tecnología Agropecuaria. Regional 8. Villavicencio, Colombia. 8 p.

Rojas, M. 1980. Manual teórico-práctico de herbicidas y fitorreguladores. Editorial: Limusa, México. 115 p.
Rzedowskii, J; Rzedowskii, G. 1979. Flora Fanerogámica del Valle de México. Ed. Continental. México. 403 p.

Rzedowskii, J; Rzedowskii, G. 2002. Flora del Bajío y de Regiones Adyacentes. Instituto de Ecología, A. C. Regional del Pátzcuaro, Michoacán. Fascículo (100). 145 p.

Sanchol, F; Cervantes, C. 1997. El uso de plantas de cobertura en sistemas de producción de cultivos perennes y anuales en Costa Rica I. Agronomía Costarricense 21(1):111-120.

SAS (Statistical Analysis Systems). 2002. SAS Versión 9.0. Institute, Inc., Cary, NC., USA.

Teasdale, R. 2005. Principios y prácticas para el uso de cultivos de cobertura en el manejo de sistemas de malezas (en línea). Consultado 13 oct. 2009. Disponible en http://www.fao.org/docrep/007/y5031s/y5031s0d.htm

Tena, M. 1998. Efecto de las prácticas de cultivo en poblaciones de arvenses y sus semillas en el suelo. Tesis Mag. Sc. Colegio de Postgraduados. Montecillos, Texcoco. México. 123 p.

Terry, P. 1996. Manejo de las malas hierbas y bananos y plátanos. In Labrada, R; Caseley, J; Parker, C. eds. Manejo de las malezas para países en desarrollo. FAO. Producción y Protección Vegetal 120: Italia, Roma. 403 p.

van Rijn, P. 2000. Weed management in the humid and subhumid tropics. Royal Tropical Institute. Amsterdam, Netherlands. 234 p.

Valentim, J; Soares, C; Alves, H; Lima, F. 2003. Velocidade de Establecimento de Acessos de Amendoim Forrageiro na Amazônia Ocidental. R. Bras. Zootec. 32(6): 1569-1577.

Vargas, A. 1997. Cultivo de banano (Musa AAA) y plátano (Musa $A A B$ ) en presencia y ausencia de una cobertura vegetal viva (Arachis pintoi CIAT-18748). CORBANA. 22(48):23-39.

Zelada E; Ibrahim, M. 1995. Tolerancia a la sombra de especies forrajeras herbáceas en el trópico húmedo de Costa Rica. Arch. Latinoam. Prod. Anim. 5 (Supl. 1):42-44. 